Planetary Systems in the Universe - Observation, Formation and Evolution

Proceedings IAU Symposium No. 202, (C)2004 IAU

Alan Penny, Pawel Artymowicz, Anne-Marie Lagrange, \& Sara Russell, eds.

\title{
Detection of Extra-solar Planets with the Keck Interferometer
}

\author{
${ }^{1}$ M.R. Swain, ${ }^{2}$ R.L. Akeson, ${ }^{1}$ M.M. Colavita \\ ${ }^{1}$ Jet Propulsion Laboratory, MS 171-113, 4800 Oak Grove, Pasadena \\ CA 91109, USA \\ ${ }^{2}$ IPAC/Caltech, MS 100-22, Pasadena CA 91125, USA
}

\begin{abstract}
We discuss the development of the "Differential Phase" detection technique for the Keck Interferometer "Hot Jupiter" Key Science Program. The goal of the "Hot Jupiter" Key Science Program is the direct detection and infrared, low-resolution spectroscopic characterization of previously detected, $\mathrm{T} \geq$ $1000 \mathrm{~K}$ extra-solar planets. Although many extra solar planets are sufficiently bright to detect with large telescopes, the proximity of these planets to the much brighter stellar primary makes direct detection difficult because of angular resolution and intensity dynamic range limitations. The Keck Interferometer, working in the near infrared, has sufficient angular resolution and sensitivity to detect some of the currently known extra solar planets; however, the intensity dynamic range required $(\sim 1: 30,000)$ is substantially beyond the capability of the traditional techniques of optical and infrared direct-detection interferometers.
\end{abstract}

\section{Introduction}

The Keck Interferometer "Hot Jupiter" Key Science program is enabled through the differential phase technique. The goals of the "Hot Jupiter" Key Science program are to (1) measure the true mass of the planet by resolving the $m$ sine $i$ ambiguity present in radial velocity measurements, (2) measure the effectively temperature of the planet, and (3) to look for spectral features, such as the predicted methane absorption line, at $\mathrm{R}=100$ in the $1.6,2.2$, and $3.8 \mu \mathrm{m}$ telluric transmission bands.

Direct detection of extra-solar planets is challenging due to the high intensity contrast and small angular separation between the planet and the star. In the infrared the system has a flux ratio of $\sim 10^{-4}$ at $2 \mu \mathrm{m}$. While the planet alone could be detected at this level, the nearby star ( $\sim 3$ mas) makes this detection a problem of intensity dynamic range and spatial resolution; the differential phase technique (Shao \& Colavita, 1992) enables the high-dynamic range phase measurements necessary to make these observations.

Although we have recently demonstrated an on-the-sky differential phase detection using the Palomar Testbed Interferometer (PTI), we have found that fluctuation in the atmospheric refractivity due to water vapor turbulence is the dominant source of error for the technique. We have explored multicolor differential phase metrics that substantially reduce the water vapor leakage term. 


\section{The Differential Phase Technique}

The differential phase technique is a multiwavelength, self-referenced phase measurement which relies on a chromatic, asymmetric brightness distribution to effectively convert a problem of intensity dynamic range into one of phase dynamic range. High precision is achieved through a differential measurement which rejects common mode errors. The goal is a differential phase sensitivity of 30 micro-radians. The differential phase technique is to measure and compare the fringe phase at two or more wave lengths. In the narrow band limit, the fringe contribution from the primary and secondary is given by

$$
V(x)=V_{p} \cos (k x)+V_{s} \cos [k(x+\delta)],
$$

where $k$ is $2 \pi / \lambda, x$ is the delay, $V_{p}$ and $V_{s}$ are the visibility amplitudes of the primary and secondary respectively, and $\delta$ is the separation of the two sources on the sky as measured in delay space and is a function of the baseline and $d s$, the separation on the sky, $\delta=\overrightarrow{d s} \cdot \vec{B}$. Both $V_{p}$ and $V_{s}$, as well as $k$ will depend on wavelength. For active fringe tracking based on quadrature measurements of the interferogram (Colavita, 1999) the relative contribution of the primary and secondary to the photon count for a particular measurement bin, A, (similarly for $\mathrm{B}, \mathrm{C}$, and $\mathrm{D})$, is given by

$$
A_{\lambda_{1}}=\int_{0}^{\lambda_{1} / 4} V_{p} \cos \left[k_{1} x\right] d x+\int_{0}^{\lambda_{1} / 4} V_{s} \cos \left[k_{1}(x+\delta)\right] d x .
$$

The simplest 2-color differential phase observable (appropriate for a non-dispersive atmosphere), $\Delta \phi$, is given by

$$
\Delta \phi=\phi_{\lambda_{1}}-\phi_{\lambda_{2}}=\tan ^{-1}\left(\frac{B_{\lambda_{1}}-D_{\lambda_{1}}}{A_{\lambda_{1}}-C_{\lambda_{1}}}\right)-\tan ^{-1}\left(\frac{B_{\lambda_{2}}-D_{\lambda_{2}}}{A_{\lambda_{2}}-C_{\lambda_{2}}}\right)
$$

\section{Atmospheric Dispersion}

Differential dispersion between the arms of the interferometer results in a nonastrophysical differential phase signature. The refractivity of the dry atmosphere is well understood in the near infrared (Owen, 1967). Atmospheric refractivity fluctuation caused by thermal and water vapor turbulence presents a challenge for the differential phase observable which must simultaneously solve for the atmospheric and astrophysical differential phase signatures. Precipitable water vapor produces most of the dispersion in the near IR.

\section{Discussion}

While fluctuations in atmospheric refractivity do present a challenge for differential phase observations we believe that a multicolor differential phase metric will permit us to solve for both the atmospheric and astrophysical differential 


\begin{tabular}{cc}
$\mathrm{K}$ Band Water Vapor Refractivity \\
\hline wavelength $(\mu \mathrm{m})$ & $\mathrm{N}(\mathrm{E}-7)$ \\
2.00 & 9.06252 \\
2.10 & 9.03147 \\
2.20 & 8.99908 \\
2.30 & 8.95824 \\
2.40 & 8.89620 \\
\hline
\end{tabular}

Table 1. Water vapor refractivity values for selected wavelengths in the $\mathrm{K}$ band. Calculated for $\mathrm{Q}=10^{17} \mathrm{~mol} / \mathrm{cc}\left(3 \mathrm{~g} / \mathrm{m}^{3}\right), \mathrm{T}=273 \mathrm{~K}$ and total pressure $\mathrm{P}=600$ mbar.

phase signatures. Using the Palomar Tested Interferometer (Colavita, 1999), we have demonstrated a differential phase detection on the sky (Akeson et al., 1999) and have demonstrated a 3-color differential phase metric which reduced the water vapor leakage by a factor of 20 .

Acknowledgments. This work was performed at the Jet Propulsion Laboratory, California Institute of Technology, under a contract with the National Aeronautics and Space Administration. Interferometer data were obtained at Palomar Observatory using the NASA Palomar Testbed Interferometer supported by NASA contracts to the Jet Propulsion Laboratory and observations at PTI are made possible through the efforts of the PTI Collaboration ( http://huey.jpl.nasa.gov/palomar/ptimembers.html ). Funding for the Keck Interferometer provided by the National Aeronautics and Space Administration.

\section{References}

Akeson, R.L., Swain, M.R., \& Colavita, M.C. 1999, A.S.P., proceedings in press

Colavita M. M. et al. 1999, ApJ, 510, 505

Colavita, M. M. 1999, PASP, 111, 111

Owens, J. C. 1967, AO, 6, 1, 51

Shao, M., \& Colavita, M.M. 1992, ARA\&A, 30, 457 